Ann. Biol. anim. Bioch. Biophys., I964, 4 (3), 263-27r.

\title{
VARIATIONS DES TAUX ENERGÉTIQUE ET AZOTÉ DANS L'ALIMENTATION DU PORC EN CROISSANCE OBSERVATIONS PRÉLIMINAIRES
}

\author{
Y. IIUNRY et A. RÉRAT \\ avec la collaboration technique de E. ENGrand et H. Bouş̨ueT \\ Station de Recherches sur lÉlerage des Porcs, \\ Centre national de Recherches zootechniques, Jouy-en-Josas (Seine-et-Oise)
}

\section{SOMMAIRE}

Deux expériences successives sont réalisées sur 88 porcs de race Large White, entre 20 et $90 \mathrm{~kg}$ de poids vif, afin d'étudier linfluence des variations des taux énergétique et azoté du régime sur la croissance, la consommation et la composition corporelle. A cet effet, on utilise des régimes semisynthétiques renfermant une source azotée unique (farine de poisson de Norvège), d'origine et de composition connues; l'augmentation du taux énergétique est obtenue en introduisant de l'huile d'arachide en remplacenent de l'amidon. I.a première expérience porte sur la comparaison de deux régimes à i $6 \mathrm{p}$. 1oo de protéines de poisson et renfermant respectivenent 5 et I $2 \mathrm{p}$. Ioo d'huile d'arachide. La deuxième comprend 4 lots, distribués suivant un schéma factoriel : 2 taux de protéines de poisson ( 16 et 8 p. Ioo) et 2 taux d'huile d'arachide ( 5 et I 5 p. I0o) correspondant respectivement à $\mathrm{I}, 2$ et $\mathrm{I}, 4$ L.F. par kg de régime. Les animaux, provenant de l'élevage expérinental de Jouy-enJosas, sont élevés en loges individuelles et nourris suivant la méthode semi ad libitum.

L'augmentation du tạx éaergé ique dı régime (de $\mathrm{r}, 2$ à $\mathrm{r}, 1$ U.F./kg environ) ne se traduit que par une faible amélioration de la vitesse de croissance $(2,5$ à $3 \mathrm{p} .100)$ entre 20 et $90 \mathrm{~kg}$ de poids vif aux deux niveaux azotés considérés ; la quantité movenne journalière d'aliment ingérée est nettement réduite, mais la consommation d'énergie est plus élevée ( $5 \mathrm{p}$. I00 en plus); les animaux sont plus gras à l'abattage, tandis que l'efficacité énergétique est la même pour les deux taux de lipides. Pour chacun des critères étudiés, il n'existe aucune interaction entre le taux azoté et le taux énergétique.

I'évolution des critères en fonction de l'âge fait apparaître une amélioration sensible de la croissance au cours des 6 premières semaines d'expérience, c'est-à-dire jusqu'à $40 \mathrm{~kg}$ de poids vif (II 5I20 jours d'âge), lorsqu'on accroît le niveau énergétique de la ration ce $\mathrm{r}, 2$ à $\mathrm{r}, 1 \mathrm{U}$.F. $/ \mathrm{kg}$. Cette amélioration est due principalement ì une augmentation notable de la consommation d'énergie, la quantité de nourriture ingérée étant au moins égale ou supérieure à celle enregistrée dans le lot témoin.

Compte tenu des observations précédentes, le jeune Porc semble répondre favorablement à un enrichissement du régime en lipides, mais il n'y a pas lieu d'utiliser ce type de régime au-delà de $40 \mathrm{~kg}$ de poids vif au risque de nuire à la cualité de la carcasse.

\section{INTRODUCTION}

Les nombreuses recherches portant sur l'étude du rapport azote-énergie dans l'alimentation des volailles (HII, I, I 962) ont montré la nécessité de réaliser un équilibre convenable entre les fractions azotée et énergétique du régime. A la suite de ces 
observations, un certain nombre d'auteurs ont étudié chez le Porc l'influence du taux énergétique sur la croissance, la consommation et la composition corporelle, grâce à l'introduction dans la ration de proportions variables de graisses alimentaires. I' une manière générale, il a été observé que les animaux qui reçoivent des régimes riches en lipicies présentent une croissance améliorée, mais sont plus gras à l'abattage (Clawson, I96I ; MoRgan et Robrnson, I962 ; LEWIs, I963). I ans certains cas, cependant, la vitesse de croissance n'est pas modifiée, pas plus que la qualité des carcasses. En réalité, cette divergence dans les observations des différents auteurs peut être attribuée pour une large part aux conditions variables d'expérimentation. En particulier, le niveau énergétique et la nature des sources énergétiques du régime diffèrent d'une expérience à l'autre ; il en est de même du taux de matières azotées et de la nature de ces dernières. De plus, la qualité des mélanges azotés utilisés présente souvent des variations d'un lot à l'autre au sein d'une même expérience, tandis que la consommation d'énergie, au lieu d'être mesurée directement, fait l'objet le plus souvent d'une simple estimation d'après les tables ou les résultats d'analyse.

Les observations précédentes nous ont conduit à étudier l'influence du rapport azote-énergie dans l'alimentation du Porc en croissance, en faisant varier les taux énergétique et azoté dans des régimes semi-synthétiques renfermant une source azotée unique, d'origine et de composition connues, et des sources énergétiques purifiées.

\section{MÉTHODES EXPÉRIMENTALES ET MATÉRIEL UTILISÉ}

Deux expériences ont été réalisées sur 88 porcs de race Large $W$ hite, entre 20 et go $\mathrm{kg}$ de poids vif, dans le but d'étudier l'influence des variations des taux énergétique et azoté du régime sur la croissance, la consommation et la composition corporelle. La source azotée utilisée est une farine de poisson de Norvège dont les caractéristiques ont été précisées dans une publication antérieure (RÉRAT et HENRY, I964). Les variations du taux énergétique sont obtenues en introduisant de l'huile d'arachide en remplacement de l'amidon.

Le schéma expérimental et la composition des régimes sont rapportés dans le tableau I. L'expérience A porte sur la comparaison de 2 régimes dont le taux protidique ( 6 p. roo de protéines de poisson) a été reconnu optimum pour la croissance dans une étude précédente (RÉRAT et HenRY, I964); ils renferment respectivement 5 et I 2 p. 100 d'huile d'arachide. L'expérience B est conçue suivant un schéma factoriel comportant :

2 taux de protéines de poisson, nettement distincts : i 6 et 8 p. 100;

2 taux d'huile d'arachide : 5 et $15 \mathrm{p}$. 100 .

Les taux azoté et énergétique, dans chacun des lots, sont maintenus constants au cours de la croissance pour faciliter l'interprétation des résultats.

Les animaux, pris au sevrage, sont mis en expérience après une période préexpérimentale de deux semaines, à un âge moyen de 74 jours, en tenant compte de l'origine, du sexe et du poids initial. Ils sont élevés en loges individuelles et reçoivent, en administration semi ad libitum, 3 repas courts de 20 à 30 minutes par jour ; ces derniers sont distribués à l'état humide, à raison de 3 parties d'eau pour une partie d'aliment. Les consommations de nourriture sont enregistrées quotidiennement et les pesées des animaux ont lieu régulièrement tous les $\mathrm{I}_{4}$ jours.

Après abattage, les carcasses sont préparées suivant la découpe parisienne. Les critères retenus pour l'étude de la composition corporelle sont : le rendement, les pourcentages de morceaux maigres (jambon + longe) et de morceaux gras (bardière + panne) dans la carcasse, l'épaisseur du lard dorsal (moyenne des mensurations au niveau du dos et du rein).

Les teneurs en énergie brute des régimes ont été déterminées à l'aide de la bombe calorimétrique. Les valeurs fourragères correspondantes, d'après les chiffres cités par FinGERLING (I953) pour l'énergie nette des différents composants, sont de l'ordre de I,2 et I,4 U.F. respectivement pour 5 et I 5 p. I00 d'huile d'arachide. 
TABLEAU I

Schéma expérimental et composition des régimes

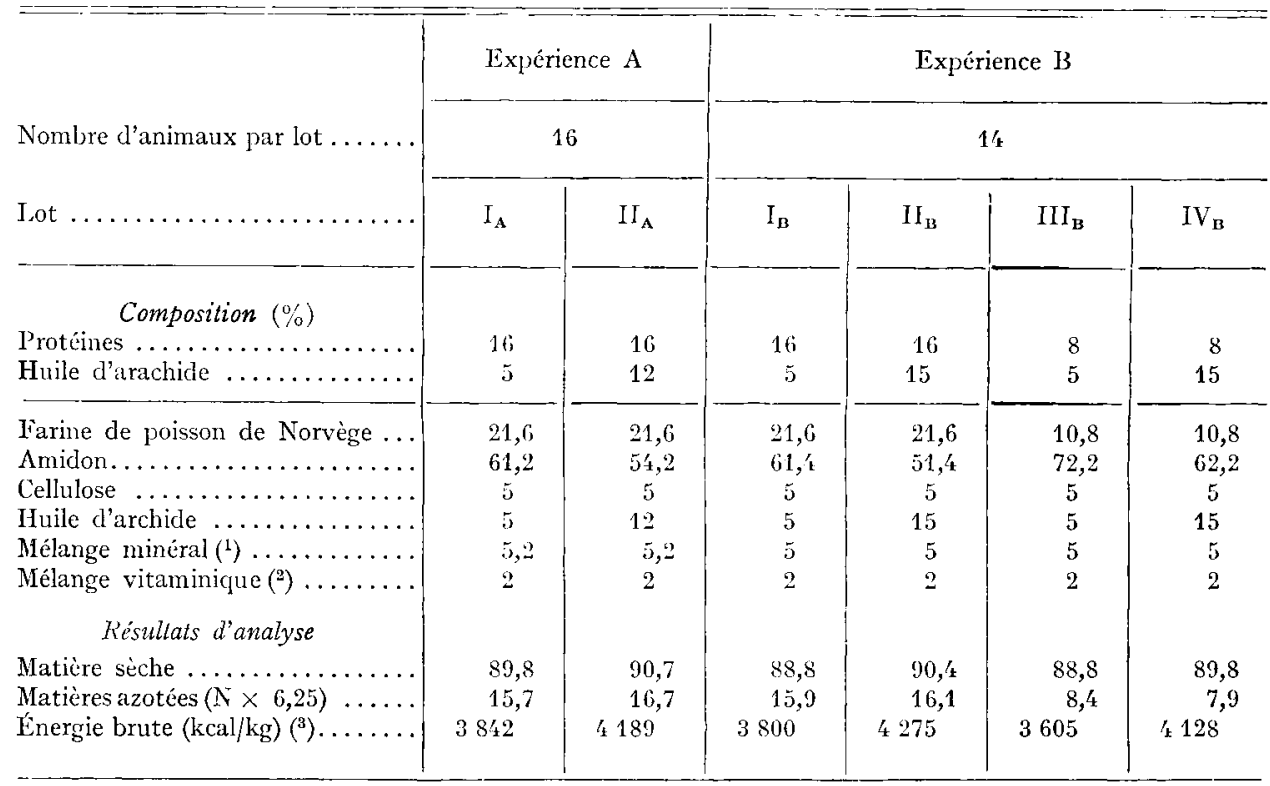

(1) Composition p. 100 : chlorure de potassium, 6,7; phosphate bicalcique, 46,3; chlorure de sodium, 13,5 ; carbonate de calcium, 16,8; carbonate de magnésium, 8,5; phosphate monopotassique, 5,7 ; sulfate ferreux, $72 \mathrm{O}, 1,5$; sulfate de manganèse, $\mathrm{H}_{2} \mathrm{O}, 0,3$; sulfate de cuivre, $5 \mathrm{H}_{2} \mathrm{O}, 0,1$; sulfate de zinc, 0,5 ; sulfate de cobalt, $7 \mathrm{H}_{2} \mathrm{O}, 0,05$; iodure de potassium, 0,05 .

$\left(^{2}\right)$ Mélange à base d'amidon apportant par $\mathrm{kg}$ de régime : thiamine, $2,2 \mathrm{mg}$; riboflavine, 6,2 $\mathrm{mg}$; niacine $22 \mathrm{mg}$; acide pantothénique, $20 \mathrm{mg}$; pyridoxine HCl, $2,6 \mathrm{mg}$; acide folique, 1,6 $\mathrm{mg}$; inositol, $0,4 \mathrm{mg}$; acide paraminobenzoïque, $4_{4}^{\prime} \mathrm{mg}$; biotine, $0,12 \mathrm{mg}$; vitamine $B_{12}, 22 \mu \mathrm{g}$; choline, $2 \mathrm{~g}$; vitamine $\mathrm{K}, 4,4_{4} \mathrm{mg}$; vitamine $\mathrm{E}$ (tocophérol), $160 \mathrm{mg}$; vitamine A, $4000 \mathrm{UI}$, vitamine $\mathrm{D}, 400 \mathrm{UI}$.

(3) Mesurée à la bombe calorimétrique.

\section{RÉSULTATS}

\section{r. - Croissance et consommation}

Les résultats généraux de croissance et de consommation, pour la période totale et pour les intervalles de poids $20-60 \mathrm{~kg}$ et $60-90 \mathrm{~kg}$, sont rapportés dans le tableau 2 . D'une manière générale, l'augmentation de la proportion d'huile d'arachide dans le régime ne provoque aucune amélioration significative de la vitesse de croissance entre 20 et $90 \mathrm{~kg}$ de poids vif, aux deux niveaux azotés considérés; il en est de même pour les périodes $20-60 \mathrm{~kg}$ et $60-90 \mathrm{~kg}$. En même temps, la consommation journalière, exprimée en $\mathrm{kg}$ d'aliment, est notablement réduite (respectivement ro et $7 \mathrm{p}$. Ioo en moins dans les expériences $A$ et $B$ sur l'ensemble de la croissance). Exprimée en énergie 


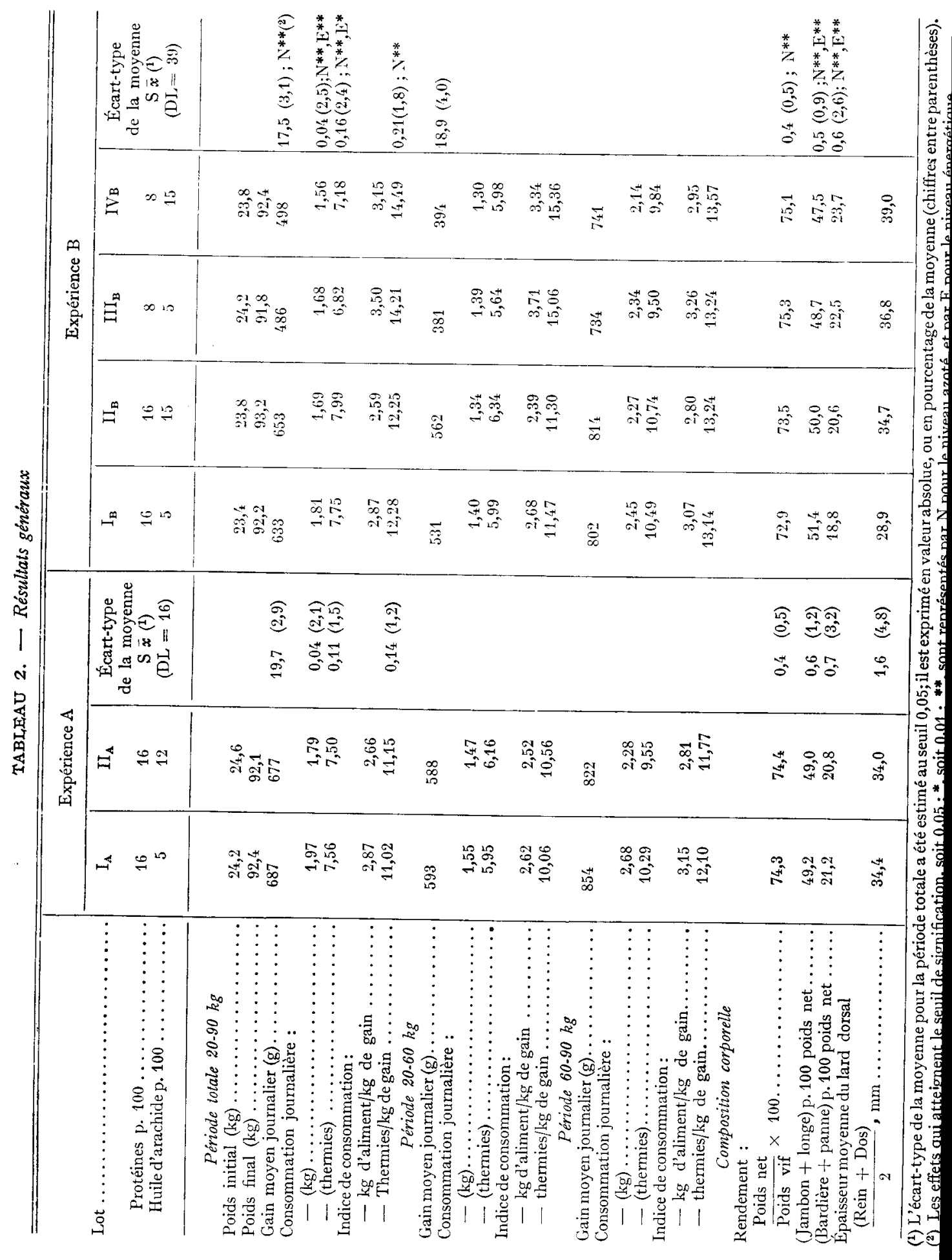




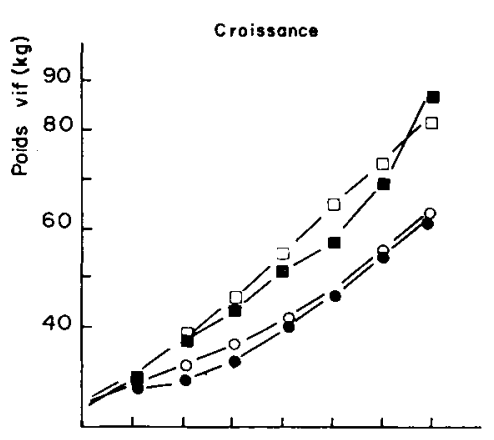

$g / \mathrm{i}$ Vitesse de croissonce $9 / \mathrm{j}$
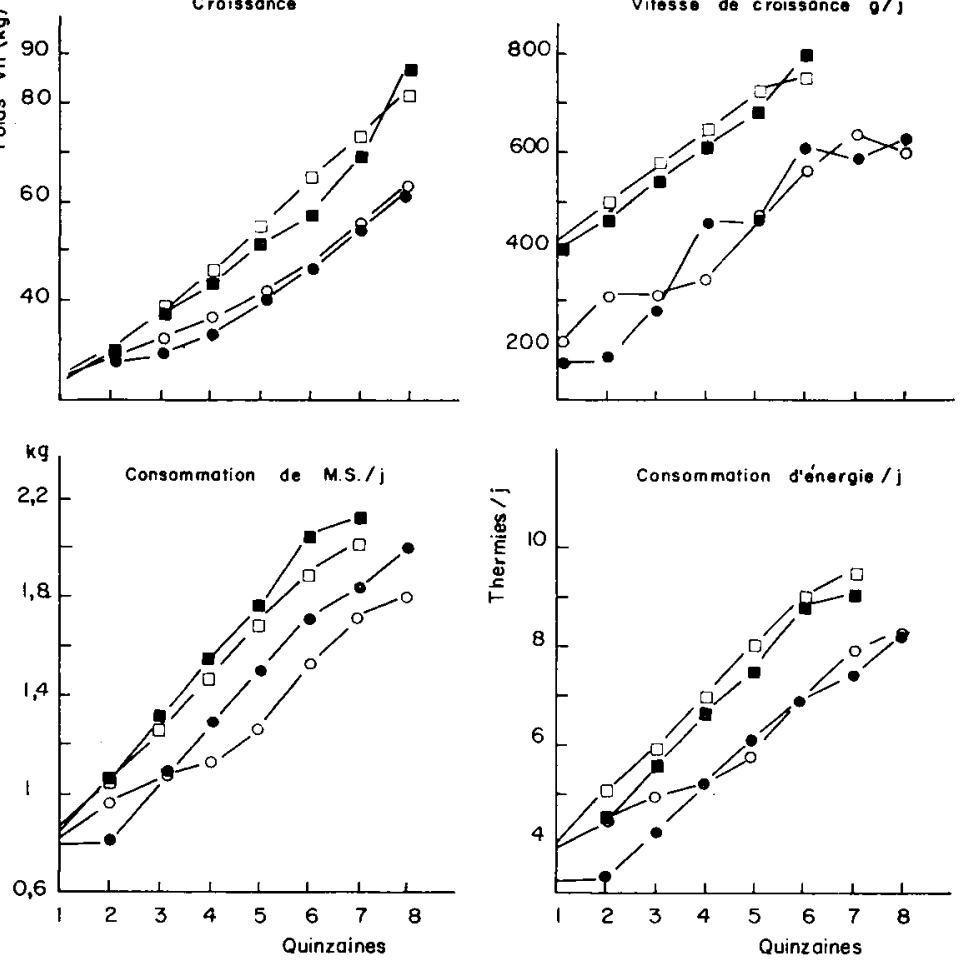

FIG. 1. - Livolution des critères au cours de la croissance (Expérience B).

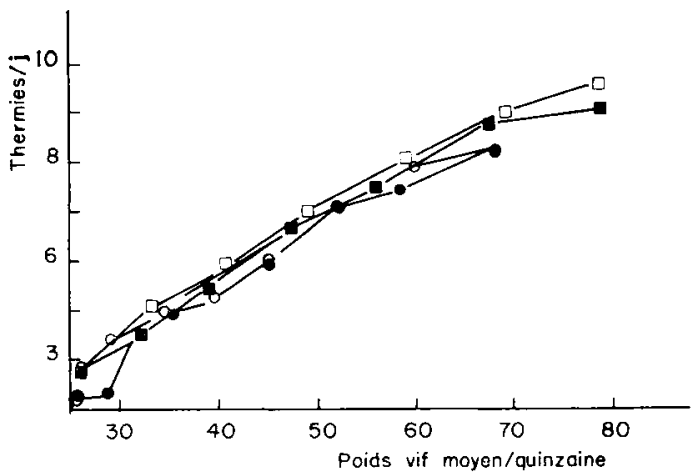

FIG. 2. - Evolution de la consommation d'énergie en foncion du poids aif (Éxpérience B):

$\begin{array}{lcc} & \begin{array}{c}\text { Lipides } \\ \text { p. } 100\end{array} & \begin{array}{c}\text { Protéines } \\ \text { P. } 100\end{array} \\ \text { - III B } & -5 & - \\ \text { O IV B } & 15 & 8 \\ \text { - I } 8 & 5 & 16 \\ \text { D II B } & 15 & 16\end{array}$

Légende des figures I et 2. 
brute, elle n'est pratiquement pas modifiée dans l'expérience A, alors que dans l'expérience $B$, pour laquelle les variations du taux énergétique sont plus importantes, elle est significativement plus élevée au niveau énergétique supérieur (respectivement 3 et 5 p. Ioo en plus pour i 6 et 8 p. Ioo de matières azotées). Dans tous les cas, la quantité d'énergie consommée par $\mathrm{kg}$ de gain est la même pour un niveau azoté donné, quelle que soit la période envisagée. L'influence đu niveau azoté est particulièrement nette pour tous les critères et similaire à celle mise en évidence lors d'expériences antérieures (RÉRAT et HENRY, Ig64).

L'évolution des critères en fonction de l'âge, décrite dans la figure $\mathrm{I}$, si elle ne fait ressortir aucune différence globale entre les lots à $60 \mathrm{~kg}$ et go $\mathrm{kg}$ de poids vif, met néanmoins en évidence une nette augmentation de la vitesse de croissance au cours des six premières semaines d'expérience chez les animaux recevant les régimes les plus énergétiques (lots $\mathrm{II}_{\mathrm{B}}$ et $\mathrm{IV}_{\mathrm{r}}$ ) ; la consommation journalière de matière sèche est pratiquement la même (régime à I 6 p. Ioo de protéines) ou supérieure (régime à 8 p. roo de protéines) ; il en résulte que la quantité d'énergie ingérée est nettement plus élevée que dans les lots témoins. L'interprétation statistique des résultats à l'issue de cette période fait d'ailleurs apparaître une influence significative du niveau énergétique sur la vitesse de croissance et la consommation d'énergie. La consommation d'énergie en fonction du poids vif est toutefois peu différente d'un lot à l'autre (fig. 2).

\section{2. - Composition corporelle (tab1. 2)}

Les variations de la composition corporelle en fonction du taux énergétique vont dans le même sens que celles de la consommation d'énergie. Ainsi, dans l'expérience $\mathrm{A}$, la composition corporelle est la même pour les deux lots, tandis que dans l'expérience $B$, les animaux recevant les régimes enrichis en lipides sont significativement plus gras que ceux des lots témoins. Par contre, l'augmentation du taux azoté entraîne une diminution de l'adiposité. De même que pour la vitesse de croissance, il n'existe aucune interaction significative entre les niveaux énergétique et azoté.

\section{3. - Influence du sexe (tab1. 3)}

Étant donné que l'expérience $\mathrm{B}$ comporte le même nombre de répétitions avec des animaux mâles et femelles, il a été procédé à l'analyse statistique de l'influence du sexe sur les différents critères ; remarquons cependant que le test $\mathrm{F}$ a été effectué par rapport à la variance résiduelle de l'effet blocs, correspondant à 12 degrés de liberté contre 39 pour la variance résiduelle estimée sur l'ensemble des données. Comme le montre le tableau 3 , les femelles présentent une vitesse de croissance significativement plus lente et une consommation d'énergie plus faible que les mâles, tandis que l'efficacité énergétique du régime est la même. Du point de vue de la composition corporelle, les femelles sont plus maigres que les mâles, mais le rendement n'est pas différent. 
TABLEAU 3

Influence du sexe. Résultats entre 20 et go $\mathrm{kg}$ de poids vif (expérience B)

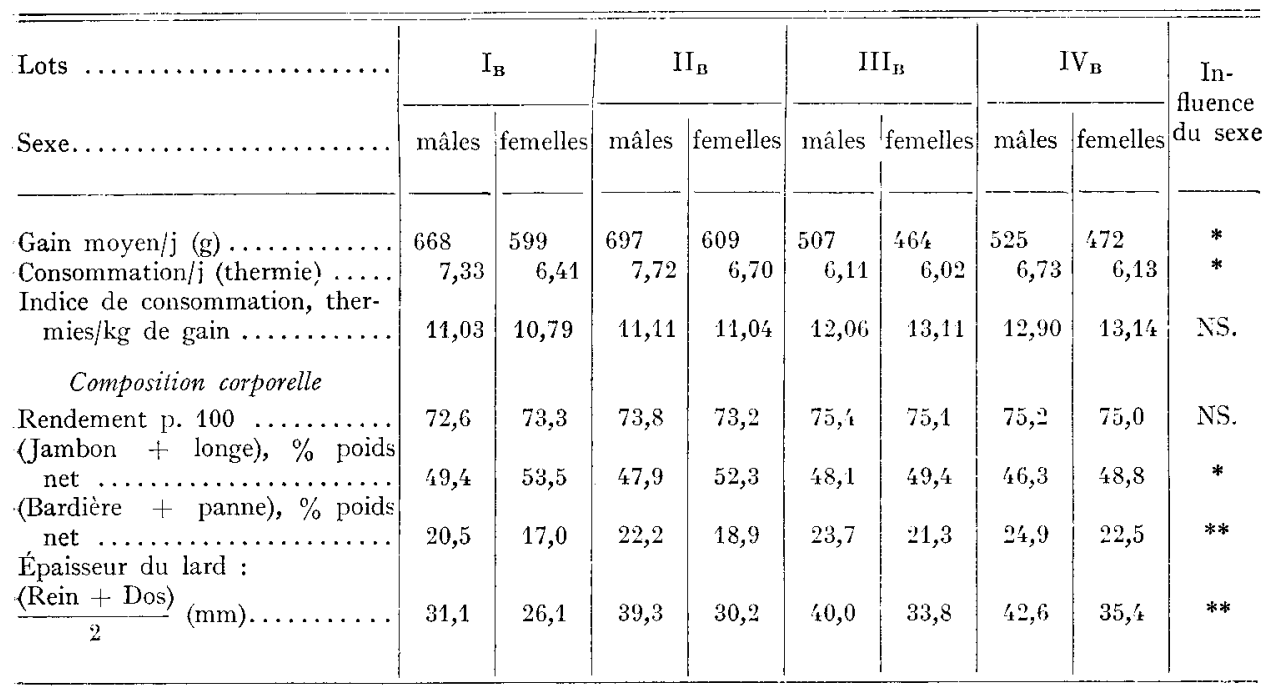

** Seuil 0,01 .

* Seuil 0,05 .

N.S. : influence non significative.

\section{DISCUSSION}

I,es résultats obtenus dans la présente étude montrent que l'introduction de lipides dans le régime provoque une amélioration faible ou insignifiante de la vitesse de croissance entre 20 et $90 \mathrm{~kg}$ de poids vif et que les animaux sont plus gras à l'abattage En réalité, cette augmentation de l'adiposité est en relation avec une consommation d'énergie accrue, comme l'ont observé par ailleurs PERRY et al. (I959) ; Bowland (Ig62) ; Hays, WaGner et Clark (I963). Ainsi, dans l'expérience B, les animaux recevant les régimes à $\mathrm{I} 5 \mathrm{p}$. Ioo d'huile d'arachide ont une croissance légèrement supérieure, une consommation d'énergie significativement plus élevée et sont nettement plus gras que ceux du lot témoin, alors que dans l'expérience A la composition corporelle est la même pour des niveaux de croissance et d'ingestion d'énergie comparables. La conséquence logique de ce qui précède est que l'indice de consommation, exprimé en énergie brute par unité de gain de poids n'est pratiquement pas modifié lorsqu'on élève le taux énergétique de la ration, puisque l'amélioration de la vitesse de croissance, lorsqu'elle existe, est accompagnée d'une augmentation du contenu énergétique du gain de poids. Remarquons, par ailleurs, que si les variations du taux énergétique n'ont qu'une influence réduite sur la vitesse de croissance, ceci 
peut être attribué à la grande richesse en énergie de tous les régimes utilisés, même le régime témoin ; les valeurs fourragères correspondantes sont ainsi comprises entre I,2 et I,4 U.F., selon le taux de lipides, contre 0,9 à I,o U.F. pour les régimes habituels, et l'amplitude de la réponse aurait été probablement plus forte si la ration de base avait été moins riche en énergie. L'influence du sexe peut s'expliquer de la même façon par une intensité de croissance plus forte et une consommation d'énergie plus élevée chez les mâles que chez les femelles.

Le fait qu'il n'y ait aucune interaction entre l'influence du taux énergétique et celle du taux azoté dans nos expériences doit être souligné. Il est en effet généralement admis que l'évolution de la croissance et de la consommation d'énergie en fonction. du taux énergétique est la même aux différents niveaux azotés, sauf toutefois lorsqu'il s'agit de régimes renfermant un faible taux de matières azotées ou des protéines de mauvaise qualité ; dans ce cas, on enregistre une dépression de la croissance consécutive à un déséquilibre entre les fractions azotée et énergétique (CLAwson et al., I962 ; Lowrey et al., I963). Dans la présente étude, au contraire, l'évolution des critères est la même pour des taux azotés très différents.

Ainsi, quel que soit le taux azoté du régime, l'addition de lipides se traduit par une légère augmentation de la vitesse de croissance et ce fait est d'autant plus sensible que l'animal est plus jeune. Dans le cas des régimes à taux élevé de protéines l'explication est simple : compte tenu de la haute valeur biologique des protéines de poisson, 1'énergie représente le facteur limitant du régime et le besoin azoté de croissance reste largement couvert, en dépit de la réduction de consommation provoquée par l'addition de lipides. Au taux azoté le plus faible, l'addition de lipides provoque au contraire, pendant six semaines, une augmentation de la consommation de matière sèche; la satisfaction du besoin azoté étant ainsi réalisée dans d'aussi bonnes conditions, il n'y a aucune raison pour que la vitesse de croissance soit ralentie.

En ce qui concerne la composition corporelle, il ne semble pas non plus qu'il existe une interaction entre le taux énergétique et le taux azoté (ou le rapport énergie/ matières azotées). Autrement dit, quel que soit le taux azoté de la ration, l'élévation du niveau énergétique provoque la formation de carcasses plus grasses. Si l'on considère l'effet propre du taux azoté, l'augmentation de ce dernier se traduit par une amélioration de la qualité des carcasses, ainsi que cela a déjà été antérieureent démontré (RÉRAT et HENRY, I964).

Enfin, un dernier point mérite d'être noté. C'est pendant son jeune âge quele Porc répond le plus favorablement à une augmentation du taux énergétique du régime et ce fait est essentiellement lié à l'augmentation de la consommation d'énergie. Cette observation confirme les travaux de divers auteurs (SEWEL, THomas et Price, I96I ; Robrison, I962). Par contre, au-delà de $40 \mathrm{~kg}$ de poids vif (correspondant à II 5-I20 j d'âge) il n'y a pas lieu d'accroître le niveau énergétique au-dessus de I,2 U.F., puisque la vitesse de croissance n'est pas différente et que les carcasses sont nettement dépréciées. D'autres études seront nécessaires pour déterminer le taux optimum d'énergie dans la ration aux différents stades physiologiques de la croissance. 


\section{SUMMARY}

\section{EFFECT OF LEVELS OF ENERGY AND PROTEIN ON PERFORMANCE AND CARCASE COMPOSITION OF GROWING-FINISHING PIGS}

Two experiments were done on 88 Large White pigs from 20 to $90 \mathrm{~kg}$ liveweight, to study the effect of different levels of energy and protein in semi-synthetic diets on growth, feed consumption and body composition. Herringmeal of known origin and composition was used as the only source of protein and energy was increased by replacing starch with groundnut oil.

In the first experiment (A), two groups of 16 pigs were given diets with 16 p. Ioo protein supplied by the fishmeal and 5 or I 2 p. Ioo groundnut oil (table I). In the second experiment (B), four groups of 14 pigs were compared according to a $2 \times 2$ factorial design : diets had two levels of protein, 16 and 8 p. I00, and two levels of groundnut oil, 5 and I 5 p. I00. The animals were alloted to groups according to litter, sex and initial weight, and were fed in individual pens. They were given as much as they could eat in 20 to 30 minutes 3 times a day.

An increase in the energy of the diet from I.2 to I.4 Scandinavian feed units per kg gave a small improvement of 2.5 to $3 \mathrm{p}$. 100 in liveweight gain during the whole period of growth (table 2) with either level of protein. The mean daily intake of feed was reduced but intake of energy was 5 p. 100 more than on the control diets. Percentage of fat in the carcase was increased. Feed efficiency, expressed as total intake of energy per unit liveweight gained, was the same at the two levels of energy. There was no interaction between levels of energy and of protein. More protein gave significantly more efficient gains and leaner carcases. The influence of sex was studied also : barrows gained significantly faster and had significantly more fat in the carcase than did gilts (table 3 ).

When we consider performance in relation to age it can be seen that the liveweight gain was significantly improved during the first six weeks of the experiments, up to II 5 to I 20 days of age and $40 \mathrm{~kg}$ liveweight (fig. I). This improvement was related to an increased intake of energy ; the amount of feed eaten was at least as much as on the control diet.

From these observations, it seems that the young pig responds favourably to an enrichment of the diet with fat, but there is no advantage from continuing such a diet beyond $40 \mathrm{~kg}$ liveweight since carcasses would be reduced in quality.

\section{RÉFÉRENCES BIBLIOGRAPHIQUES}

Bowland J. P., 1962. Levels and ratios of energy and protein in swine rations. Feedstuffs, 34, I3, 30-32. Clawson A. J., I96r. Calorie-protein ratios for growing pigs. Feedsiuffs, $33(4), 36$.

Clawson A. J., Blumer T. N., Smart W. W. G. jr, Barrick E. R., i 962 . Influence of energy-protein ratio on performance and carcass characteristics of swine. J. Anim. Sci. 21, 62-68.

FINGERLING G., 1953. Die Respirationsversuche mit verschiedenen Futtermitteln, in NeHRING K., WeRNER A., Untersuchungen über den Stärkewert Verschiedener Futtermittel. Band I, 25-326. Deutsche Akademie der Landwirtschaftwissenschaften, Berlin.

HAYS V. W., WAGNeR G. R., CLARK A. J., I963. Effect of energy and protein levels on performance and carcass characteristics of pigs. Feedsiuffs, $\mathbf{3 5}(25), 28$.

Hill F. W., I962. Some aspects of the physiology of food intake and digestion in chickens, in MorgAN J. T., LEWIS A., Nutrition of pigs and poultry, 3-7, Butterworth, London.

LEwIS D., I963. Basic factors influencing the efficiency of utilization of energy and protein with special reference to their interrelationships. Confévence mondiale de Zootechnie, Rapports généraux, vol. I., 305342, Rome..

Lowrey R. S., Pond W. G., Loosli J. K., BARnes R. H., I 963 . Effect of dietary protein and fat on growth, protein utilization and carcass composition of pigs fed purified diets. J. Anim. Sci., 22, I09-I I4.

MoRgan J. T., Robinson D. W., 1952. Dietary factors and the performance of growing-finishing pigs., in Morgan J. T., Lewis D. Nutrition of pigs and Poultry, Butterworth, London, 255-285.

Perry T. W., Kennington M. H., Beeson .W. M., 1952. The addition of animal fat to swine rations. Feedstuffs, 31 (7), 26-27.

Rérat A., Henry Y., ig64. Étude du besoin azoté chez le Porc en croissance. I. Utilisation de la farine de poisson à trois taux différents. Ann. Zootech., 13, 5-34.

Sewell R. F., Thomas M. C., Price D., 196r. Protein-energy relatonships in the rations of earlyweaned pigs. J. Anim. Sci., 20., 820-823.

WAGNer G. R., Clark A. J., HaYs V. W., SPEer V. C., 1963. Effect of protein-energy relationships on the performance and carcass quality of growing swine. J. Anim. Sci, 22, 202-208. 